\title{
Effect of high-BDNF microenvironment stem cells therapy on neurogenic bladder model in rats
}

\author{
Wen Jie Tian ${ }^{1,2}$, Seung Hwan Jeon ${ }^{1,2}$, Guan Qun Zhu ${ }^{3}$, Eun Bi Kwon ${ }^{2}$, Ga Eun Kim², Woong Jin Bae ${ }^{1,2}$, \\ Hyuk Jin Cho ${ }^{1}$, U-Syn $\mathrm{Ha}^{1}$, Sung-Hoo Hong ${ }^{1}$, Ji Youl Lee ${ }^{1}$, Kang Sup Kim ${ }^{1}$, Sae Woong Kim ${ }^{1,2}$ \\ ${ }^{1}$ Department of Urology, College of Medicine, The Catholic University of Korea, Seoul, Republic of Korea; ${ }^{2}$ Catholic Integrative Medicine Research \\ Institute, the Catholic University of Korea, Seoul, Republic of Korea; ${ }^{3}$ Department of Urology, Affiliated Hospital of Qingdao University, Qingdao, \\ China \\ Contributions: (I) Conception and design: WJ Tian, SH Jeon, WJ Bae; (II) Administrative support: SH Jeon; (III) Provision of study materials or \\ patients: All authors; (IV) Collection and assembly of data: WJ Tian, SH Jeon, SW Kim; (V) Data analysis and interpretation: WJ Tian, SH Jeon, \\ SW Kim; (VI) Manuscript writing: All authors; (VII) Final approval of manuscript: All authors. \\ Correspondence to: Sae Woong Kim, MD, PhD. Department of Urology, College of Medicine, The Catholic University of Korea, 222, Banpo-daero, \\ Seocho-gu, Seoul 06591, Republic of Korea. Email: ksw1227@catholic.ac.kr.
}

Background: The purpose of this study is to explore the effects of high-BDNF microenvironment produced by engineered immortalized mesenchymal stem cells (imMSCs) on the neurogenic bladder (NB) and investigate underlying mechanism.

Methods: Male Sprague-Dawley rat (12-week-old, weighing about 370-400 g) were purchased from a Korean company (Orient Bio Co. Seongnam, Korea) and divided into the following groups (n=32): sham control group ( $n=8)$, NB group ( $n=8), N B+\operatorname{ImMSCs}$ group ( $=8), N B+\operatorname{ImMSCs}($ BDNF) group ( $=8$ ). The major pelvic ganglion (MPG) was observed under anesthesia. Three NB groups of rats were then subjected to bilateral MPG injury. The sham control group of rats was treated with sham surgery. Cystometry were performed before the rats were sacrificed, and then MPG and bladder were collected for histochemical and Western blot analysis.

Results: MSCs treatment improves lower urinary tract function, and the NB + ImMSCs (BDNF) group is better than the NB + ImMSCs group $(\mathrm{P}<0.01)$. MSCs treatment accelerates recovery of injured nerve tissue, and the NB + ImMSCs (BDNF) group is better than the NB + ImMSCs group $(\mathrm{P}<0.01)$. In high BDNF environment, apoptosis was reduced more significantly and muscle tissue recovered more rapidly $(\mathrm{P}<0.01)$. High-BDNF microenvironment activates more BDNF/TrkB/CREB signaling pathways $(\mathrm{P}<0.01)$.

Conclusions: In a rat NB model caused by nerve injury, imMSCs have certain effects on nerve tissue repair. At the same time, it was proved that increasing the expression of BDNF which had specific effect on nerve injury repair could more effectively repair injured MPG in local microenvironment. The mechanism may be related to the activation of the BDNF/TrkB/CREB signaling pathway and the reduction of apoptosis by highly expressed BDNF.

Keywords: Neurogenic bladder (NB); immortalized mesenchymal stem cells; high BDNF microenvironment; upgraded BDNF engineered stem cells; BDNF/TrkB/CREB pathway

Submitted Jul 07, 2020. Accepted for publication Nov 04, 2020.

doi: $10.21037 /$ tau-20-1072

View this article at: http://dx.doi.org/10.21037/tau-20-1072

\footnotetext{
^ ORCID: 0000-0002-6037-1319.
} 


\section{Introduction}

Bladder and urethral dysfunction caused by damage to the nervous system that controls urination is called neurogenic bladder (NB). Some causes of NB include diabetes, cerebrovascular accidents, brain injury, spinal cord injury, and nerve injury. Resulting urinary complications such as urinary tract infection, upper urinary tract damage, and renal failure are main causes of death (1). Although there are ways to prevent or delay NB complications, research into restoring the bladder itself is still in its infancy (2).

Stem cells have the ability to repair various tissues, and researchers have used them in various ways to treat various diseases (3-6). Mesenchymal stem cells (MSCs) are pluripotent stem cells with all characteristics of stem cells, namely self-renewal, multifunction, and multidirectional differentiation. Nowadays, in many medical fields, stem cell therapy has been applied clinically, achieving satisfactory results $(7,8)$. Our previous study (9) has shown that MSCs treatment can accelerate the recovery of damaged nerves and tissues. Therefore, it is reasonable to believe that MSCs treatment can show a good effect in NB treatment. In order to improve the proliferation ability and anti-aging ability of stem cells, more researchers $(10,11)$ using immortalized mesenchymal stem cells (imMSC) which is more stable compared to MSCs.

Brain-derived neuronutrition factor (BDNF) is a neurotrophin (NT). Some researchers have found that stable expression of BDNF in progenitor cells can effectively repair damaged nerve tissues (12). Researchers found that BDNF also significantly increased the cross-sectional area of the muscle innervated by the corresponding nerve $(13,14)$. Therefore, we used gene technology to increase the expression of BDNF in MSCs to observe the effect of locally high BDNF microenvironment on therapeutic effect in a neurogenic bladder model of rats caused by nerve injury, and to compare the therapeutic effect of local high BDNF microenvironment with that of general MSCs, confirming that the local high BDNF microenvironment has better therapeutic effect.

In this study, we used an NB model of pelvic ganglion injury in rats and studied treatment with engineered MSCs. We hypothesized that when we increased the expression of BDNF in MSCs, we could construct a locally high-BDNF microenvironment to improve the repair ability of nerve tissue and more effectively treat neurogenic bladder in rats caused by nerve injury. We present the following article in accordance with the ARRIVE reporting checklist (available at http://dx.doi.org/10.21037/tau-20-1072).

\section{Method}

\section{High BDNF expressing engineered MSCs preparation}

Primary bone marrow mesenchymal stem cells (BMMSCs) were cultured in low glucose-containing Dulbecco's modified Eagle's medium (DMEM, Gibco, US) supplemented with $20 \%$ fetal bovine serum (FBS, Gibco, US) and $5 \mathrm{ng} / \mathrm{mL}$ basis fibroblast growth factor (bFGF, Cell Signaling Technology, Danvers, MA, USA) at $37^{\circ} \mathrm{C}$ with $5 \%$ $\mathrm{CO}_{2}$, but engineered BM-MSCs were cultured with $10 \%$ FBS. To generate engineered hTERT, BM-MSCs, c-myc, and tetracycline transactivator ( $\mathrm{tTA}$ ) and BDNF genes were synthesized and transfected with pBD lentiviral vector (SL BIGEN, Incheon, Korea). In brief, the reference sequence of BDNF is NP_733927.1 and we prepared optimized DNA sequence to introduce into a vector (Genscript, Piscataway, NJ, USA). DNA sequencing was performed to confirm whether the right reading frame of the introduced sequence is correct (Cosmogenetech, Seoul, Korea). The cryopreserved cells were thawed and inoculated 12well plates, at a density of $5 \times 10^{5}$ cells per well. 48 hours after, the supernatant was harvested and BDNF protein level was determined. The qualified expression level of BDNF was about $10 \mathrm{ng} / \mathrm{ml}$. Transfected engineered BMMSCs [imMSCs (BDNF)] were selected monoclonal by antibiotics. Selected imMSCs (BDNF) were isolated by limited dilution. Blank carrier were administered into BMMSCs (imMSCs) as a control. The final monoclonal cell was selected by BDNF protein expression, proliferation rate and other MSC phenotypes. The engineered imMSCs was irradiated before injection in vivo (Since immortalized stem cells have possibility that cause tumors in vivo, we irradiated these cells before administration to stop proliferation).

\section{ELISA}

Inoculate BM-MSCs imMSCs and imMSCs (BDNF) into $100 \mathrm{~mm}$ petri dishes. After complete adhesion, the complete growth medium was removed and the cells were washed with PBS for 3 times. Subsequently, $10 \mathrm{~mL}$ of fresh serumfree basal medium was added and incubated at $37{ }^{\circ} \mathrm{C}$ with $5 \% \mathrm{CO}_{2}$ for 3 days. The collected culture medium was centrifuged at $4^{\circ} \mathrm{C}$ for 15 minutes at 3,000 rpm. Collected supernatant, imMSCs and imMSCs (BDNF) conditioned media $(\mathrm{CM})$ and test $\mathrm{BDNF}$ for each group according to 
ELISA (R\&D Systems Europe, Abingdon, UK) method. The absorbance was read on a 450-nm micrometer (Synergy H1 M, BioTek, Woburn, MA, USA). Each clone tested at least 3 separate petri dish cells.

\section{Experimental animal and animal grouping}

Experiments were performed under a project license (NO. CUMC-2016-0220-01) granted by Committee of the Catholic University of Korea, in compliance with institutional Animal Care and Use Committee (IACUC) of Catholic University of Korea for the care and use of animals. Male Sprague-Dawley rat (12-week-old, weighing about 370-400 g) were purchased from a Korean company (Orient Bio Co. Gyeonggi-do, Korea) and randomly divided into the following groups $(\mathrm{n}=32)$ : sham control group ( $\mathrm{n}=8)$, NB group $(\mathrm{n}=8), \mathrm{NB}+\operatorname{ImMSCs}$ group $(\mathrm{n}=8)$, $\mathrm{NB}+$ ImMSCs (BDNF) group $(\mathrm{n}=8)$. The major pelvic ganglion (MPG) was observed under anesthesia as described previously (15). Three NB groups of rats were then subjected to bilateral MPG injury. The sham control group of rats was treated with sham surgery. After operation, rats were kept alone and provided free access to food and water.

\section{MSCs treatment}

In groups NB + ImMSCs and NB + ImMSCs (BDNF), two kinds of imMSCs $\left(1 \times 10^{6}\right.$ MSCs diluted with phosphatebuffered saline) were injected into the smooth muscle of the bladder wall near PN on both sides under anesthesia 1 week after NB induction $(15,16)$. Rats in the NB group were injected with the same amount of normal saline. To track the location of injected imMSCs, they are labeled with fluorescent dyes (Cell Tracker ${ }^{\mathrm{TM}}$ CM-DiI; Molecular Probes, Eugene, OR, USA), according to the manufacturer's protocol.

\section{Cystometry}

All rats were subjected to a predetermined cystometry on the fourth day after treatment (17). The rats were anesthetized by subcutaneous injection of $1.2 \mathrm{mg} / \mathrm{kg}$ urethane. The bladder was exposed by suprapubic midline upper laparotomy and a 25 -gauge needle attached to a polyethylene tube was inserted through the dome of the bladder. The tube was connected through a 3 -way valve to a pressure sansor and a Harvard syringe pump to record intravesical pressure and inject saline into the bladder.
After emptying the bladder, cystometry was measured by using a saline infusion at a rate of $0.04 \mathrm{~mL} / \mathrm{min}$. Contraction interval and contraction pressure (maximum bladder pressure during urination) were recorded using a polygraph (Grass 7D; Grass Institute Co, Quincy, MA, USA). Nonvoiding contraction (NVC) was determined 4 to 2 minutes before each voiding contraction. NVC was defined as the contraction of $>4 \mathrm{cmH}_{2} \mathrm{O}$ compared to baseline pressure during bladder filling. After cystometry, MPG, Pelvic Nerve (PN) and bladder tissue were collected for histochemistry and western blot analysis.

\section{Immunofluorescent staining}

Collected MPG and PN, and bladder were fixed at $4{ }^{\circ} \mathrm{C}$ in $4 \%$ paraformaldehyde for 24 hours to make paraffin blocks. The primary antibodies used were as follows: $\beta \mathrm{III}$-tubulin (diluted 1:200; Abcam, Cambridge, MA, USA), brainderived neurotrophic factor (BDNF diluted 1:100; Abcam, Cambridge, MA, USA), poly-ADP-ribose polymerase (PARP, diluted 1:500; Abcam, Cambridge, MA, USA) were used. 6-diamidino-2-phenylindole (DAPI; Vector Laboratories, Inc., Burlingame, CA, USA) was used to stain nuclei. The digital images were obtained by using Zeiss LSM 510 Meta confocal microscope (Zeiss, Oberkochen, Germany). Mean intensity was calculated using ZEN 2012 (Zeiss).

\section{Western blot}

Collected tissue was homogenized using ice-cold RIPA buffer (Cell Signaling Technology, Danvers, MA, USA) containing ethylene diamine tetra acetic acid-free protease inhibitor cocktail and phosphatase inhibitor cocktail (Roche Diagnostics $\mathrm{GmbH}$ ). Particulate mass was removed by centrifugation at $15,000 \mathrm{~g}$ for $15 \mathrm{~min}$ at $4{ }^{\circ} \mathrm{C}$. Supernatants were analyzed by SDS-PAGE. Primary antibodies included BDNF (diluted 1:1000; Abcam, Cambridge, MA, USA), TrkB (diluted 1:1000; Abcam, Cambridge, MA, USA), p-TrkB (diluted 1:1000; Cell Signaling Technology, Danvers, MA, USA), CREB (diluted 1:500; Abcam, Cambridge, MA, USA), p-CREB (diluted 1:500; Abcam, Cambridge, MA, USA), AKT (diluted 1:200; Cell Signaling Technology, Danvers, MA, USA), p-AKT (diluted 1:200; Cell Signaling Technology, Danvers, MA, USA), Caspase-3 (diluted 1:500; Abcam, Cambridge, MA, USA), $\alpha$-smooth muscle actin (diluted 1:500; Abcam, Cambridge, MA, USA), and $\beta$-actin (diluted 1:1000; Abcam, Cambridge, MA, USA). 
A

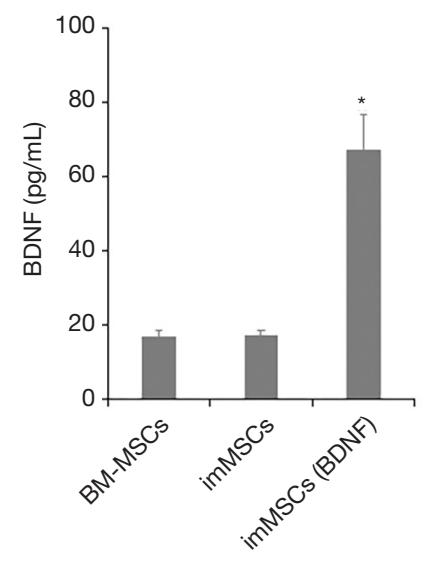

B

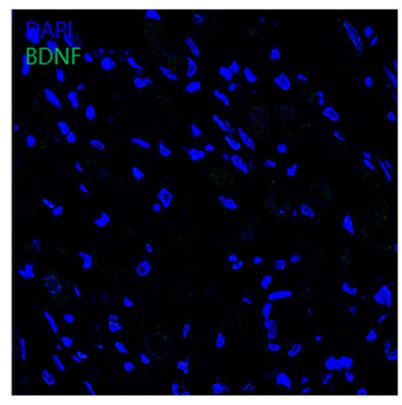

Sham control

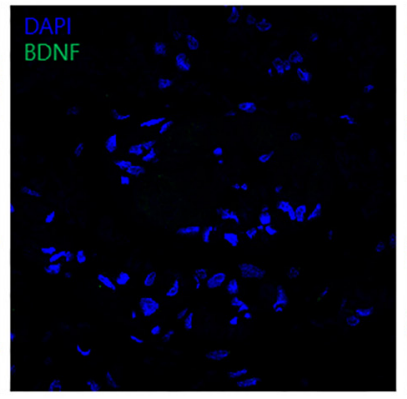

NB

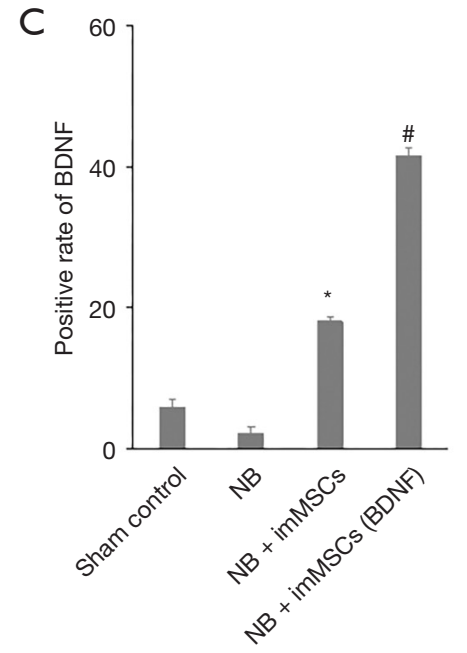

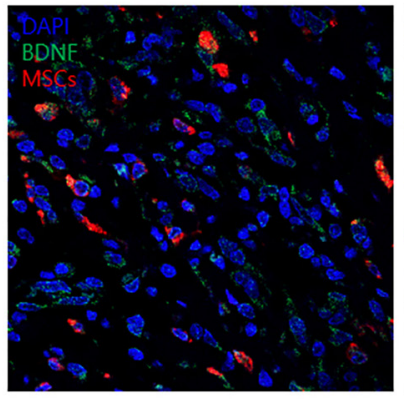

$\mathrm{NB}+\mathrm{imMSCs}$

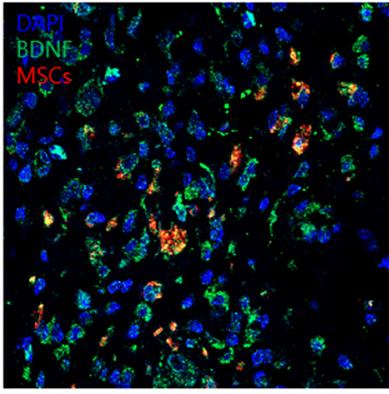

$\mathrm{NB}+$ imMSCs (BDNF)

Figure 1 the imMSCs (BDNF) make a higher BDNF microenvironment locally, both in vivo and in vitro. (A)BDNF expression in each group was determined by ELISA. *, $\mathrm{P}<0.01$ vs. BM-MSCs and imMSCs. (B) Representative images of BDNF (green color) staining and MSCs (red color) in MPG for each group. Original magnification: $\times 200$. (C)The expression of BDNF in each group was quantitatively analyzed. *, $\mathrm{P}<0.01$ vs. NB group. \#, $\mathrm{P}<0.01$ vs. $\mathrm{NB}+\mathrm{ImMSCs}$ group. Sham control is the normal control group. NB group was induced into neurogenic bladder group. NB + ImMSCs (BDNF) is for injected high BDNF expressing engineered MSCs, and imMSCs is for injected engineered MSCs transfected by empty vectors.

\section{Statistical analysis}

All data are presented as mean \pm standard error (SD). They were analyzed with SPSS version 22.0 software (IBM, Armonk, NY, USA). Student's $t$-test, one-way ANOVA, and $2 \times 2$ factorial ANOVA as appropriate were used to evaluate whether differences among groups were significant. $\mathrm{P}<0.05$ was considered statistically significant.

\section{Results}

\section{Engineered MSCs express more BDNF in vitro and in vivo}

We observed the expression of BDNF in different groups, determined the local high-BDNF microenvironment during animal experiments, and detected the expression of BDNF in vivo and in vitro. BDNF concentration in the supernatant was measured in vitro before cell injection. As can be seen from Figure 1A, ELISA results showed that imMSCs (BDNF) did increase BDNF expression in vitro $(\mathrm{P}<0.01)$. From Figure 1B, we found that the expression of BDNF in NB ( $n=8)$ group was the lowest in the tissues. BDNF expression levels of the NB + ImMSCs $(n=8)$ and $\mathrm{NB}+\operatorname{ImMSCs}(\mathrm{BDNF})(\mathrm{n}=8)$ groups are significantly higher than NB group, and NB + ImMSCs (BDNF) groups are significantly higher than $\mathrm{NB}+\mathrm{ImMSCs}$ group $(\mathrm{P}<0.01)$. This is also shown in the quantitative results in Figure $1 C$. The results showed that the imMSCs (BDNF) locally produced a higher BDNF microenvironment in vivo than 

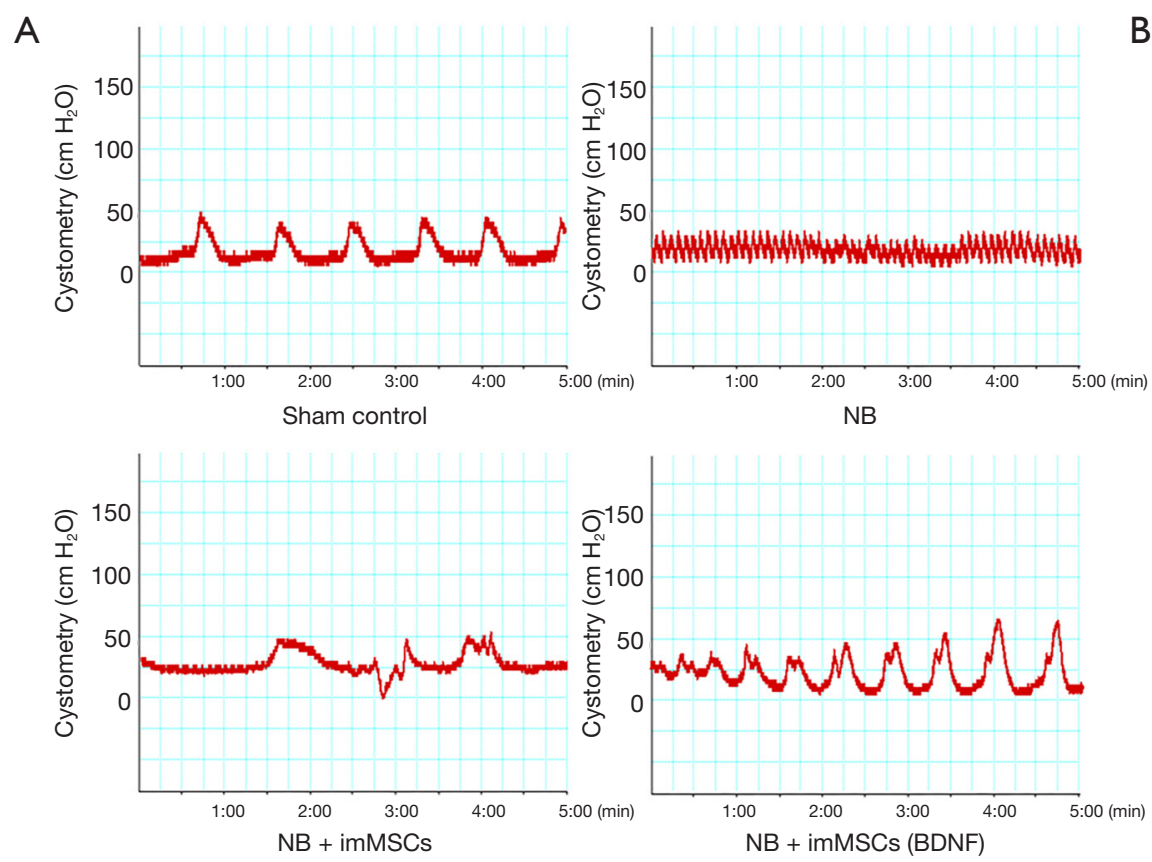

B

Figure 2 Representative images of cystometrography in each group. (A) Cystometry (H2O) for each group. (B) Each bar shows the mean contraction interval (standard deviation). * $\mathrm{P}<0.01$ vs. BM-MSCs and imMSCs. * $\mathrm{P}<0.01$ vs. NB group. \#, $\mathrm{P}<0.01$ vs. NB + ImMSCs group. Sham control is the normal control group. NB group was induced into neurogenic bladder group. NB + ImMSCs (BDNF) is for injected high BDNF expressing engineered MSCs, and imMSCs is for injected engineered MSCs transfected by empty vectors.

the empty vector transfected imMSCs.

\section{MSCs treatment improves NB function}

Bladder function was measured by Cystometry. As can be seen from Figure 2, the mean contraction interval of $\mathrm{NB}$ group was longer than other groups $(\mathrm{P}<0.01)$. As shown in Figure $2 B$, the mean contraction interval of the $\mathrm{NB}+$ ImMSCs (BDNF) $(\mathrm{n}=8)$ and NB + ImMSCs $(\mathrm{n}=8)$ groups is significantly shorter than that of the NB $(n=8)$ group $(\mathrm{P}<0.01)$, and the $\mathrm{NB}+\mathrm{ImMSCs}(\mathrm{BDNF})$ group is shorter than the $\mathrm{NB}+\mathrm{ImMSCs}$ group $(\mathrm{P}<0.01)$. The results show that imMSCs treatment can effectively improve NB function, especially in high-BDNF environment.

\section{MSCs treatment accelerates recovery of injured nerve tissue in MPG}

MPG and PN were collected and nerve recovery was detected. Quantitative analysis of Figure 3 shows that compared with the NB $(\mathrm{n}=8)$ group, $\mathrm{NB}+\mathrm{ImMSCs}(\mathrm{BDNF})$ $(\mathrm{n}=8)$ and NB + ImMSCs $(\mathrm{n}=8)$ groups have significant neurological recovery $(\mathrm{P}<0.01)$, and the recovery rate of the $\mathrm{NB}+\mathrm{ImMSCs}(\mathrm{BDNF})$ group is higher than NB + ImMSCs group $(\mathrm{P}<0.01)$. Combined with Figure $1 A$, we believe that imMSCs (BDNF) express more BDNF, forming a higher-BDNF microenvironment around nerve injury and accelerating nerve recovery.

\section{In high BDNF environments, apoptosis was reduced and muscle tissue recovery accelerated}

In the NB model, due to the injury in MPG, we studied the apoptosis of MPG in each group by PARP and Caspase-3 detection, and observed the muscle tissue recovery in the group by $\alpha$-smooth muscle actin detection. The Figure $4 A, B, C, D$ showed a few apoptosis of MPG cells in sham Control $(n=8)$ group. without treatment the apoptosis in the damaged MPG is still high $(\mathrm{P}<0.01)$. But after imMSCs injection the apoptosis was significantly reduced and the $\mathrm{NB}+\operatorname{ImMSCs}(\mathrm{BDNF})(\mathrm{n}=8)$ group was lower than $\mathrm{NB}$ $+\operatorname{ImMSCs}(\mathrm{n}=8)$ group $(\mathrm{P}<0.01)$. The Figure $4 E$ showed NB $(n=8)$ group's muscle tissue expression is significantly reduced $(\mathrm{P}<0.01)$, but after imMSCs injection the muscle 


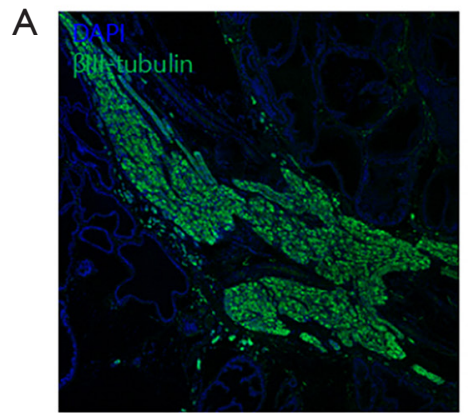

Sham control

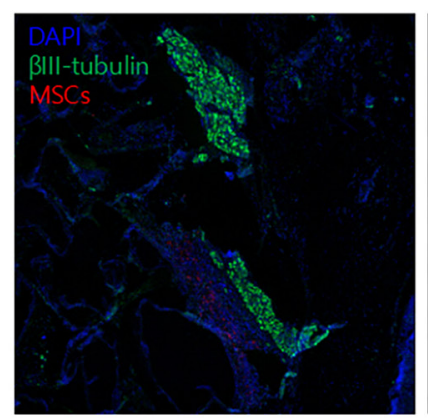

$\mathrm{NB}+$ imMSCs

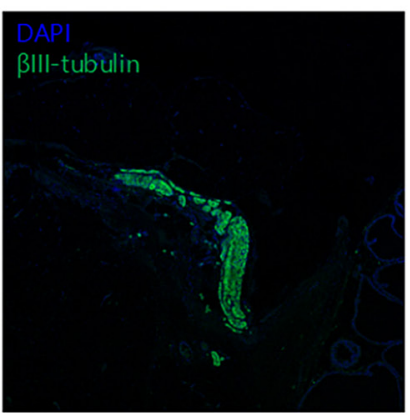

NB

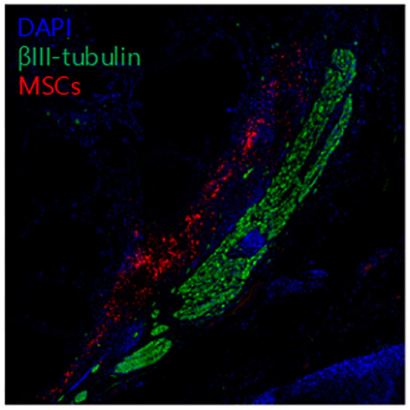

$\mathrm{NB}+$ imMSCs $(\mathrm{BDNF})$
B

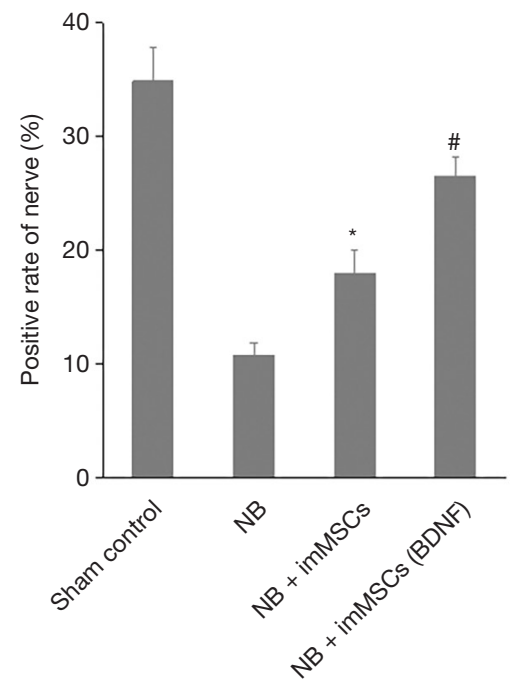

Figure 3 MSCs repair injured PN. (A) Representative images of PN staining. Green color (beta3 tubulin) is nerve, blue color is cell nucleus and red color is MSCs (Original magnification: $\times 200$ ). (B) quantitative analysis to detect the positive rate. * $\mathrm{P}<0.01$ vs. BM-MSCs and imMSCs. *, $\mathrm{P}<0.01$ vs. NB group. \#, $\mathrm{P}<0.01$ vs. NB + ImMSCs group. Sham control is the normal control group. NB group was induced into neurogenic bladder group. NB + ImMSCs (BDNF) is for injected high BDNF expressing engineered MSCs, and imMSCs is for injected engineered MSCs transfected by empty vectors.

tissue is significantly increased $(\mathrm{P}<0.01)$, and the $\mathrm{NB}+$ ImMSCs $(B D N F)(n=8)$ group recovers faster than the $\mathrm{NB}+\operatorname{ImMSCs}(\mathrm{n}=8)$ group $(\mathrm{P}<0.05)$. This suggests that apoptosis is significantly reduced and muscle tissue recovery is accelerated in high BDNF microenvironments.

\section{High BDNF microenvironment activates the BDNF/TrkB/ CREB signaling patbway}

We continue to explore the mechanisms behind it. Enhancement of the BDNF/TrkB/CREB signaling pathway is an important factor in neural tissue recovery. BDNF expression, TrkB, Akt, and CREB phosphorylation levels were measured in the extracted tissue. As shown in Figure $5 A, \mathrm{BDNF}$ expression was the same as that in Figure 1, while phosphorylation levels of TrkB, Akt and CREB were significantly reduced in the $\mathrm{BN}(\mathrm{n}=8)$ group $(\mathrm{P}<0.01)$. After imMSCs treatment, the phosphorylation level was significantly increased, and the NB + ImMSCs (BDNF) $(n=8)$ was significantly higher than the NB + ImMSCs $(\mathrm{n}=8)$ group $(\mathrm{P}<0.01)$. This is also shown in the quantitative results in Figure $5 B$. It was suggested that high-BDNF microenvironment could enhance the BDNF/TrkB/CREB signaling pathway around damaged nerves to play a role in nerve repair.

\section{Discussion}

In recent years, with development of surgical techniques, postoperative survival rate of pelvic tumor has been improved. The number of pelvic surgeries, including radical prostatectomy, rectal surgery, and hysterectomy, has been increasing year by year. Many of these patients could be injured by MPG, causing NB and affecting their quality of life $(18,19)$. Although there are many ways to alleviate complications caused by NB, we need to fundamentally treat NB to solve the problem of bladder tissue and functional recovery.

In this study, NB rat models were first established, and then engineered stem cells were injected into rats 


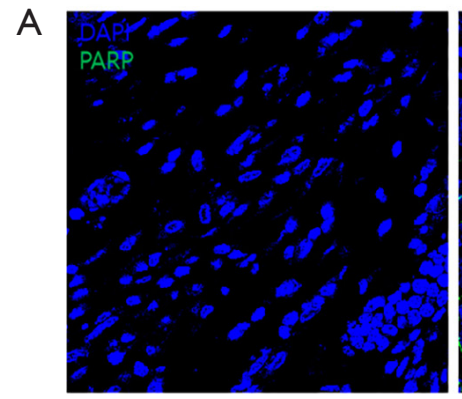

Sham control

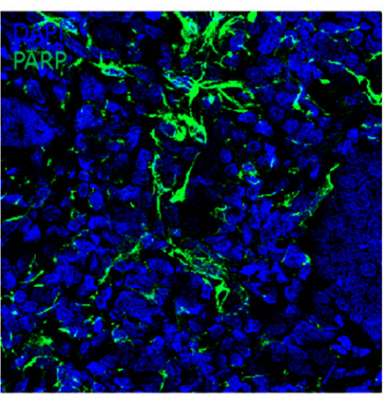

NB

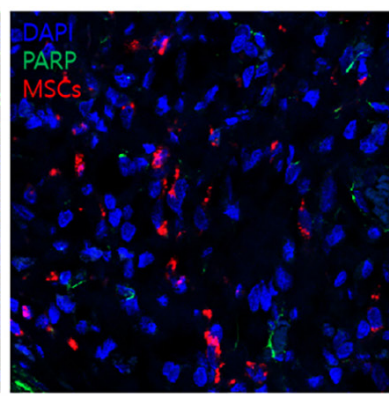

$\mathrm{NB}+\mathrm{imMSCs}$

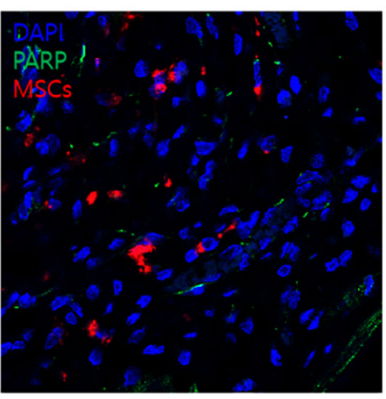

$\mathrm{NB}+\mathrm{imMSCs}(\mathrm{BDNF})$

B

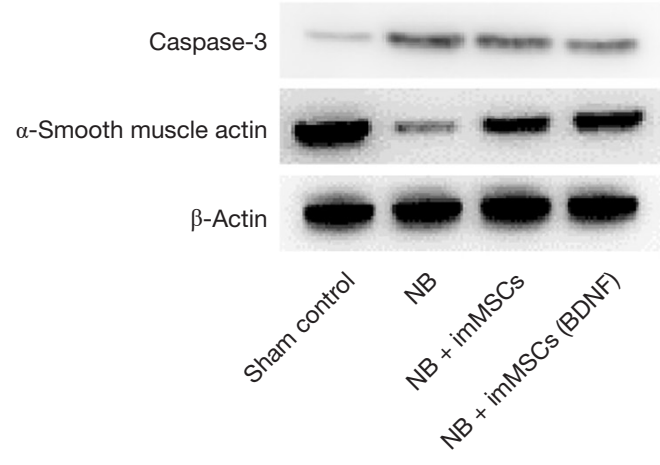

C

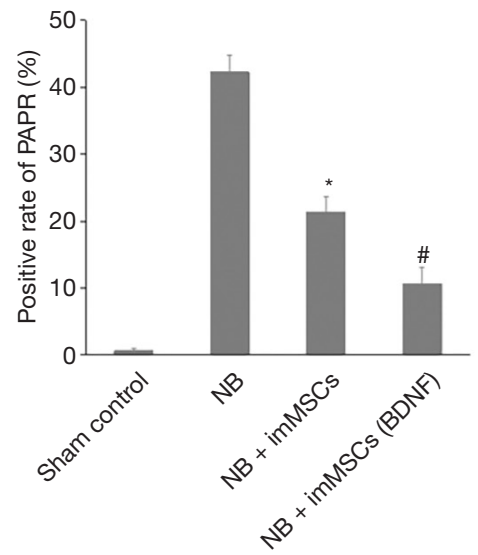

D

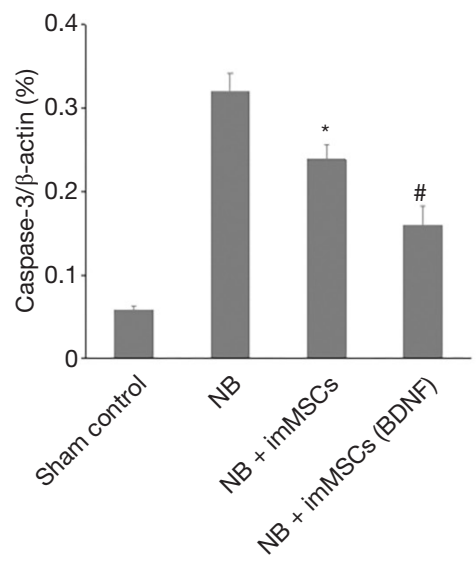

E

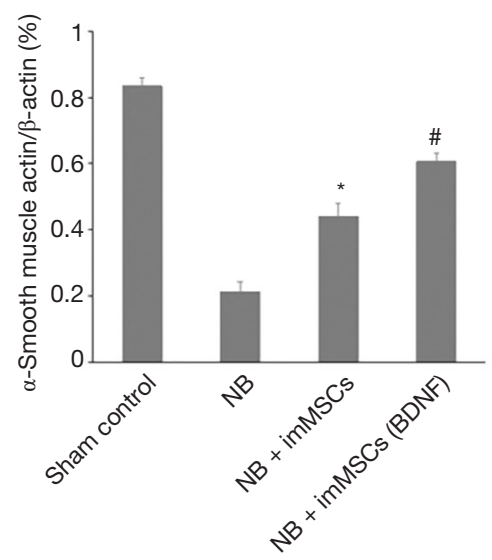

Figure 4 MSCs degrade apoptosis and Recovery of muscle tissue by increasing the expression of BDNF. (A) Representative images of apoptosis for each group. Green color is PARP, blue color is cell nucleus and red color is MSCs (Original magnification: $\times 200$ ). (B)All groups were compared for caspase- 3 and $\alpha$-smooth muscle actin in MPG by western blot. (C) Positive rate of PARP for each group was quantitatively analyzed. Each bar shows the mean values (standard deviation). (D) Western blot quantitative analysis of caspase-3/ $\beta$-actin. (E) Western blot quantitative analysis of $\alpha$-smooth muscle actin/ $\beta$-actin. Sham control is the normal control group. NB group was induced into neurogenic bladder group. NB + ImMSCs (BDNF) is for injected high BDNF expressing engineered MSCs, and imMSCs is for injected engineered MSCs transfected by empty vectors. *, $\mathrm{P}<0.01$ vs. NB group. \#, $\mathrm{P}<0.01$ vs. NB + ImMSCs group. 
A

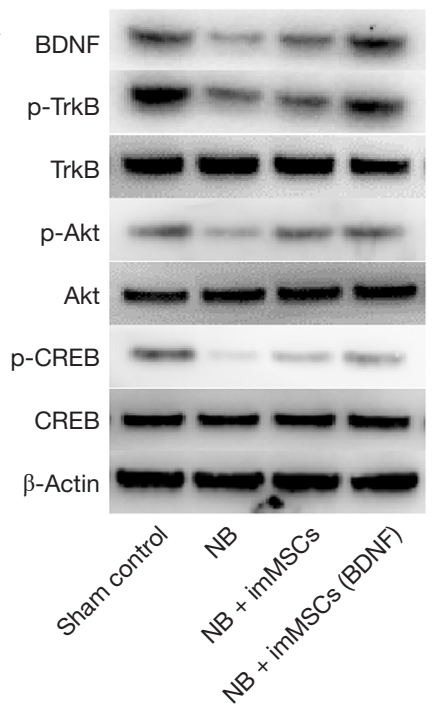

B

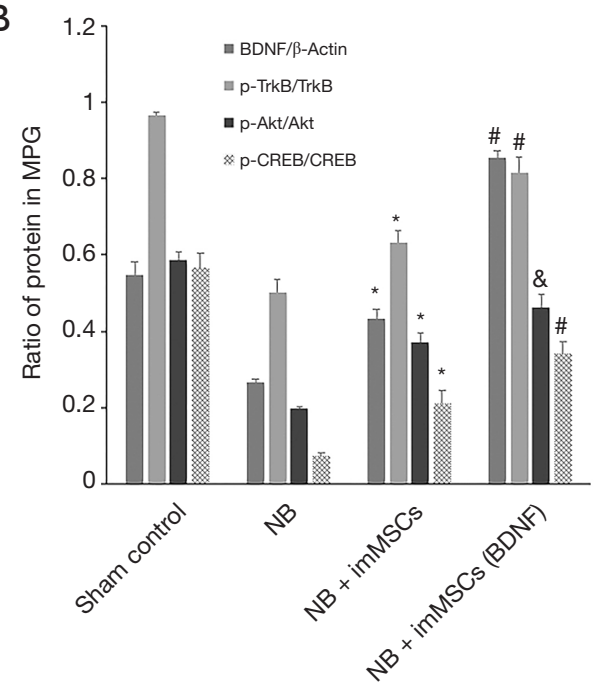

Figure 5 Effects of BDNF/TrkB/CREB signaling pathways in each group. (A) Each groups were detected for BDNF, p-TrkB, TrkB, p-Akt, Akt, p-CREB and CREB in MPG by western blot. (B) Quantity analysis of western blot, including BDNF/ $\beta$-actin, p-TrkB/TrkB, p-Akt/ Akt, p-CREB/CREB. *, $\mathrm{P}<0.05$ vs. NB group. \#, $\mathrm{P}<0.01$ vs. NB + ImMSCs group. \&, $\mathrm{P}<0.05$ vs. NB + ImMSCs group. Sham control is the normal control group. NB group was induced into neurogenic bladder group. NB + ImMSCs (BDNF) is for injected high BDNF expressing engineered MSCs, and imMSCs is for injected engineered MSCs transfected by empty vectors.

for treatment. MSCs repair tissue functions by clustering around damaged tissues. However, some researchers (20) believe that reactive oxygen species (ROS) are toxic to injected stem cells. Induction of apoptosis is the main mechanism of ROS-induced cytotoxicity, which can rapidly reduce MSCs. As a result, the number of MSCs in target tissues continues to decrease, thus reducing its effect. We used immortalized mesenchymal stem cells to avoid apoptosis of stem cells. This can provide an innate and stable microenvironment (21). We used a gene transfer technique that allowed engineered imMSCs to express more BDNF than normal imMSCs. We found that after injection of imMSCs, the average bladder internal pressure of NB rats was significantly increased and the expression of $\alpha$-SMA in bladder was significantly increased. At the same time, we found that in the NB rat model, the more BDNF around the nerve injured by MPG, the better the recovery of nerve and bladder muscle tissue, and the reduction of apoptosis. These results showed that imMSCs had good effects on function recovery and tissue repair of neurogenic bladder, while local high-BDNF expression could obtain better results.

BDNF and nerve growth factor (NGF) are both neurotrophic factor proteins. In studies on the treatment of strabismus (14), continuous treatment with BDNF resulted in slow myofibers had larger diameters, larger neuromuscular junctions, and there was increased percentage of myofibers positive for slow $\mathrm{MyHC}$ at the proximal end of the treated muscles. We continue to study its mechanism, BDNF is a key regulatory factor in the repair of nerve tissue damage. It binds to the high-affinity receptor TrkB, leading to phosphorylation of Akt and CREB, which protects nerve cells from oxidative stress and plays a role in repair (22-27). This is consistent with our results in the study. Activated $\operatorname{TrkB}$ interacts with and phosphorylates several intracellular targets (such as PI3K/ Akt, Ras/MAPK/ERK, and CaM signaling pathways) (28). Activated Akt phosphorylates its substrate and affects the activity of many kinases. Activation of CREB which important nuclear transcription factor, appears to be a key step in the signaling cascade that leads to structural changes behind the development of long-term memory (29). Interestingly, although BDNF induces CREB activation by activating Akt and produces persistent CREB signaling, the CREB signaling loop is ultimately involved in controlling the induction of BDNF (30). In addition, BDNF and NGF were found to regulate nuclear factor- $\kappa \mathrm{B}(\mathrm{NF}-\kappa \mathrm{B})$ by inhibiting neuroinflammation (31). Other studies have shown that activation of $\mathrm{BDNF} / \operatorname{TrkB}$ is involved in inhibition of the Bax/Bcl-2 ratio (a typical apoptotic index) 
after brain Ischemia/Reperfusion injury (32). In addition, inhibition of TrkB phosphorylation increases the Bax/Bcl-2 ratio, which further activates Caspase-3 (33). Although these factors confirm the role of the BDNF/TrkB/CREB signaling pathway in neuroprotection and repair, there are still many results that we have not presented and need further study.

In this study, MSCs were injected into the bladder tissue for treatment. We consider that it is difficult to directly contact the drugs around MPG when MSCs are used in clinical therapy. Therefore, we can inject imMSCs in this way to treat neural bladder and hope that this method will be clinically useful. In addition, some researchers have found that the application of microenergy in vitro can activate the proliferation and differentiation of in vivo stem cells to repair damaged muscle tissue (34). We can also use this research method for reference in the next research. There is certainly room for improvement in our research. First, due to the lack of time and efficiency, we did not detect the effect of the entire inflection point on the damaged nerve and bladder wall. In other words, the timing of the use of MSCs is still unclear, and whether another injection of MSCs is needed to achieve the best results remains to be studied. We try to prolong the treatment. However, the surgical area of rats would adhere, seriously affecting bladder measurement and tissue collection. Secondly, we only used the common MSCs concentration, and the optimal injection concentration is still uncertain. Although the concentration and duration of MSCs injection were not studied, we tested it and obtained good results. Finally, in this report we did an animal experiment. In cell experiments, we did not study the signaling pathways of MSCs to repair damaged nerve and bladder tissues. Therefore, in the following research, we will establish a co-culture platform of nerve cells and MSCs to study the effects of mesenchymal stem cells on neural proliferation and repair.

\section{Conclusions}

In a rat NB model caused by nerve injury, imMSCs have certain effects on nerve tissue repair. They have positive effects on function recovery and tissue repair of neurogenic bladder. At the same time, it was proved that increasing the expression of BDNF which had specific effect on nerve injury repair could more effectively repair injured MPG in local microenvironment. The mechanism may be related to the activation of the BDNF/TrkB/CREB signaling pathway and the reduction of apoptosis by highly expressed BDNF. These trials not only provide new ideas for NB treatment, but also provide a more effective way to use stem cells.

\section{Acknowledgments}

The authors wish to acknowledge the financial support of the Catholic Medical Center Research Foundation made in the program year of 2019.

Funding: This research was supported by Basic Science Research Program through the National Research Foundation of Korea (NRF) funded by the Ministry of Education (2020R1I1A1A01074732). This work was supported by the National Research Foundation of Korea (NRF) grant funded by the Korea government (MSIT) (No.2019R1G1A1100670).

\section{Footnote}

Reporting Checklist: The authors have completed the ARRIVE reporting checklist. Available at http://dx.doi. org/10.21037/tau-20-1072

Data Sharing Statement: Available at http://dx.doi. org/10.21037/tau-20-1072

Peer Review File: Available at http://dx.doi.org/10.21037/ tau-20-1072

Conflicts of Interest: All authors have completed the ICMJE uniform disclosure form (available at http://dx.doi. org/10.21037/tau-20-1072). All authors report grants from National Research Foundation of Korea funded by the Ministry of Education (2020R1I1A1A01074732), grants from National Research Foundation of Korea (NRF) funded by the Korea government (MSIT) (No. 2019R1G1A1100670), during the conduct of the study. The authors wish to acknowledge the financial support of the Catholic Medical Center Research Foundation made in the program year of 2019 .

Ethical Statement: The authors are accountable for all aspects of the work in ensuring that questions related to the accuracy or integrity of any part of the work are appropriately investigated and resolved. Experiments were performed under a project license (NO. CUMC-20160220-01) granted by Committee of the Catholic University of Korea, in compliance with institutional Animal Care and 
Use Committee (IACUC) of Catholic University of Korea for the care and use of animals.

Open Access Statement: This is an Open Access article distributed in accordance with the Creative Commons Attribution-NonCommercial-NoDerivs 4.0 International License (CC BY-NC-ND 4.0), which permits the noncommercial replication and distribution of the article with the strict proviso that no changes or edits are made and the original work is properly cited (including links to both the formal publication through the relevant DOI and the license). See: https://creativecommons.org/licenses/by-nc-nd/4.0/.

\section{References}

1. Ginsberg D. The epidemiology and pathophysiology of neurogenic bladder. Am J Manag Care 2013;19:s191-6.

2. Peter T. Dorsher, Peter M. McIntosh. Neurogenic Bladder. Adv Urol 2012;2012:816274.

3. Rafii S, Lyden D. Therapeutic stem and progenitor cell transplantation for organ vascularization and regeneration. Nat Med 2003;9:702-12.

4. Mackie AR, Losordo DW. CD34-positive stem cells in the treatment of heart and vascular disease in human beings. Tex Heart Inst J 2011;38:474-85.

5. Park SS. Cell therapy applications: Retinal vascular diseases-Diabetic retinopathy and retinal vein occlusion. Invest. Ophthalmol Vis Sci 2016;57 ORSF.

6. Jeon SH, Kim HY, Kim SW, et al. Engineered Mesenchymal Stem Cells Expressing Stromal Cell-derived Factor-1 Improve Erectile Dysfunction in StreptozotocinInduced Diabetic Rats.Int J Mol Sci 2018;19:3730.

7. Matthes SM, Reimers K, Radtke C, et al. Intravenous transplantation of mesenchymal stromal cells to enhance peripheral nerve regeneration. Biomed Res Int 2013;2013:573169.

8. Honmou O, Onodera R, Kocsis JD, et al. Mesenchymal stem cells: therapeutic outlook for stroke Trends Mol Med 2012;18:292-7.

9. Zhu GQ, Jeon SH, Kim SW, et al. Efficient Promotion of Autophagy and Angiogenesis Using Mesenchymal Stem Cell Therapy Enhanced by the Low-Energy Shock Waves in the Treatment of Erectile Dysfunction. Stem Cells Int 2018;2018:1302672.

10. Liu TM, Ng WM, Tan HS, et al. Molecular basis of immortalization of human mesenchymal stem cells by combination of $\mathrm{p} 53$ knockdown and human telomerase reverse transcriptase overexpression Stem Cells Dev
2013;22:268-78.

11. Gong M, Bi Y, Li T, et al. Immortalized mesenchymal stem cells: an alternative to primary mesenchymal stem cells in neuronal differentiation and neuroregeneration associated studies J Biomed Sci 2011;18:87.

12. Butenschön J, Zimmermann T, Leschik J, et al. PSANCAM positive neural progenitors stably expressing BDNF promote functional recovery in a mouse model of spinal cord injury. Stem Cell Res Ther 2016;7:11.

13. Nelson KR, Stevens SM, McLoon LK. Prolongation of Relaxation Time in Extraocular Muscles With Brain Derived Neurotrophic Factor in Adult Rabbit. Invest Ophthalmol Vis Sci 2016;57:5834-42.

14. Christy L. Willoughby, Jérome Fleuriet, et al.Adaptation of Slow Myofibers: The Effect of Sustained BDNF Treatment of Extraocular Muscles in Infant Nonhuman Primates. Invest Ophthalmol Vis Sci 2015;56:3467-83.

15. Jeon SH, Shrestha KR, Lee JY, et al. Combination Therapy Using Human Adipose-derived Stem Cells on the Cavernous Nerve and Low-energy Shockwaves on the Corpus Cavernosum in a Rat Model of Post-prostatectomy Erectile Dysfunction. Urology 2016;88:226.e1-9.

16. Zhu GQ, Jeon SH, Bae WJ, et al. Engineered Stem Cells Improve Neurogenic Bladder by Overexpressing SDF1 in a Pelvic Nerve Injury Rat Model. Cell Transplant 2020;29:963689720902466.

17. R. Soler, C. Füllhase, C. Santos, et al. Development of bladder dysfunction in a rat model of dopaminergic brain lesion. Neurourol Urodyn 2011;30:188.

18. Groen J, Pannek J, Castro Diaz D, et al. Summary of European Association of Urology (EAU) Guidelines on Neuro-Urology. Eur Urol 2016;69:324-33.

19. Eveno C, Lamblin A, Mariette C, et al. Sexual and urinary dysfunction after proctectomy for rectal cancer. J Visc Surg 2010;147:e21-30.

20. Liu GY, Jiang XX, Zhu X, et al. ROS activates JNKmediated autophagy to counteract apoptosis in mouse mesenchymal stem cells in vitro. Acta Pharmacol Sin 2015;36:1473-9.

21. Liu MC, Chen WH, Chiou CS, et al. Inhibition of chronic prostate inflammation by hyaluronic acid through an immortalized human prostate stromal cell line model. PLoS One 2017;12:e0178152.

22. Ai J, Sun L. H, Che H, et al. MicroRNA-195 protects against dementia induced by chronic brain hypoperfusion via its anti-amyloidogenic effect in rats. J Neurosci 2013;33:3989-4001. 
23. Xu Q, Ji XF, Chi T, et al. Sigma 1 receptor activation regulates brain-derived neurotrophic factor through NR2A-CaMKIV-TORC1 pathway to rescue the impairment of learning and memory induced by brain ischaemia/reperfusion. Psychopharmacology 2015;232:1779-91.

24. Pringle AK, Sundstrom LE, Wilde GJ, et al. Brain-derived neurotrophic factor, but not neurotrophin-3, prevents ischaemia-induced neuronal cell death in organotypic rat hippocampal slice cultures. Neurosci Lett 1996;211:203-6.

25. Almeida RD, Manadas BJ, Melo CV. Neuroprotection by BDNF against glutamate-induced apoptotic cell death is mediated by ERK and PI3-kinase pathways. Cell Death Differ 2005;12:1329-43.

26. Rössler OG, Giehl KM, Thiel G. Neuroprotection of immortalized hippocampal neurones by brainderived neurotrophic factor and Raf-1 protein kinase: role of extracellular signal-regulated protein kinase and phosphatidylinositol 3-kinase. J Neurochem 2004;88:1240-52.

27. Yoo JM, Lee BD, Kim MR, et al. Neuroprotective action of $\mathrm{N}$-acetyl serotonin in oxidative stress-induced apoptosis through the activation of both TrkB/CREB/BDNF pathway and Akt/Nrf2/Antioxidant enzyme in neuronal cells. Redox Biol 2017;11:592-9.

Cite this article as: Tian WJ, Jeon SH, Zhu GQ, Kwon EB, Kim GE, Bae WJ, Cho HJ, Ha US, Hong SH, Lee JY, Kim KS, Kim SW. Effect of high-BDNF microenvironment stem cells therapy on neurogenic bladder model in rats. Transl Androl Urol 2021;10(1):345-355. doi: 10.21037/tau-20-1072
28. Segal RA, Greenberg ME. Intracellular signaling pathways activated by neurotrophic factors. Annu Rev Neurosci 1996;19:463-89.

29. Bourtchuladze R, Frenguelli B, Blendy J, et al. Deficient long-term memory in mice with a targeted mutation of the cAMP-responsive element-binding protein. Cell 1994;79:59-68.

30. Bramham CR, Messaoudi E. BDNF function in adult synaptic plasticity: the synaptic consolidation hypothesis. Prog Neurobiol 2005;76:99-125.

31. Cai H, Wang Y, He J, et al. Neuroprotective effects of bajijiasu against cognitive impairment induced by amyloid- $\beta$ in APP/PS1 mice. Oncotarget 2017;8:92621-34.

32. Zhao Y, Wang JL, Deng B, et al. TAT-Ngn2 Enhances Cognitive Function Recovery and Regulates CaspaseDependent and Mitochondrial Apoptotic Pathways After Experimental Stroke. Front Cell Neurosci 2018;12:475.

33. Cui K, Kang N, Lue T, et al. Microenergy acoustic pulses induced myogenesis of urethral striated muscle stem/ progenitor cells. Transl Androl Urol 2019;8:489-500.

34. Yang B, Li M, Lei H, et al. Low Intensity Pulsed Ultrasound Influences the Myogenic Differentiation of Muscle Satellite Cells in a Stress Urinary Incontinence Rat Model. Urology 2019;123:297.e1-297.e8. 